\title{
Methodology of the Global and Regional Burden of Stroke Study
}

\author{
The Global Burden of Disease Stroke Expert Group
}

\section{Key Words}

Stroke - Disability - Systematic review - Epidemiology •

Global health $\cdot$ Methodology

\begin{abstract}
Background: Setting priorities for the prevention of stroke requires an empirical understanding of the pattern of disease burden and exposure to major risk factors. In this manuscript we aim to report the methodology of a systematic review of the epidemiological literature on stroke and how this information will be synthesized to produce updated estimates of the global burden of stroke. Methods: We will use multi-state models implemented in the software program DisMod III to estimate age-specific prevalence, incidence, and early case-fatality (defined as either 28-day, 30-day or 1-month case fatality) for stroke by the 21 global burden of disease (GBD) regions as well as by gender and pathological stroke type based on information obtained from a systematic review. We conducted a two-stage search strategy in order to identify studies published between 1980 and 2011 for the GBD stroke review. Eligible studies: (a) distinguished between stroke and transient ischaemic attack (TIA); (b) distinguished between 1st ever and recurrent stroke; (c) reported on age-specific rates; (d) if reported, provided survival status within 28 days, 30 days or 1 month of onset for fatal and nonfatal events; (e) specified methods for ascertaining stroke cases, and (f) described imaging modalities to determine
\end{abstract}

stroke subtypes. Details of included studies were recorded on a detailed data extraction form by trained reviewers. We will gather information on demographics, natural history and clinical outcomes (e.g. Rankin scale, Glasgow Coma Scale), after stroke which will be used to facilitate the estimation of epidemiological parameters. Reporting and methodological quality was rated. Populations were coded as urban, rural, or combined and studies classified as national, subnational, healthcare system-based, or community level. Studies published in non-English languages were translated and coded centrally. Discussion: In international health research, there is a crucial need for accurate assessment of global health patterns. A thorough GBD reassessment of stroke will ensure that global health policy decisions are based on the most up-to-date, valid and reliable epidemiological information available.

Copyright $\odot 2011$ S. Karger AG, Basel

\section{Background}

Cerebrovascular diseases, principally stroke, can be prevented to a large extent as there are several modifiable risk factors (including high blood pressure, smoking, lack of physical activity and an unhealthy diet), accounting for $90 \%$ of the risk of stroke [1]. Stroke is the second leading cause of death worldwide [2], and caused an estimated 5.7 million deaths in 2005 and the number of

\section{KARGER}

Fax +41613061234 E-Mail karger@karger.ch www.karger.com
(C) 2011 S. Karger AG, Basel

0251-5350/12/0381-0030\$38.00/0

Accessible online at:

www.karger.com/ned
Derrick A. Bennett

Clinical Trials Service Unit, Richard Doll Building, Old Road Campus

Roosevelt Drive

Oxford OX3 7LF (UK)

Tel. +44 1865 743949, E-Mail derrick.bennett@ctsu.ox.ac.uk 
global deaths is projected to rise to 6.5 million in 2015 and 7.8 million in 2030 [2]. Stroke is not predominantly a disease of the oldest old in high-income countries, given that of the 5.7 million people who died of stroke in 2005 , fewer than 1.0 million lived in high-income countries and about $40 \%$ of stroke victims were less than 70 years of age [2]. Although rates of stroke mortality and morbidity differ greatly between countries, the overall burden of stroke is greater in low-income (where the incidence and case fatality are rising) than in high-income countries $[3,4]$.

Setting priorities for the prevention of stroke requires an empirical understanding of the pattern of disease and exposure to major risk factors $[5,6]$. The original Global Burden of Diseases, Injuries, and Risk Factors (GBD) Study that began in 1990 used epidemiological information systematically collected in collaboration with scientific experts from around the world to identify a comprehensive and standardized set of measures of global health encompassing communicable diseases, non-communicable diseases, and injuries [7]. The original project created a common metric - the Disability-Adjusted Life Year (DALY) - to generate comparable information on disease burden for regions that collectively spans the world's population. Two decades have passed since the original GBD epidemiological reviews were conducted. During that time, periodic reassessments of the global burden of disease procedures have been made, with improvements in data availability and methods for dealing with incomplete and biased data [8]. A new round of the GBD Study has now been convened with approximately 40 expert groups to conduct systematic reviews, covering the period 1980 to 2011, of studies and available data to estimate incidence, prevalence, case fatality, and cause specific mortality for each disease, injury, and risk factor [9]. Original estimates are to be revised given the new data and improved methods, and new estimates generated. This iteration of the study will present estimates in 21 GBD Study geographic regions [10] of the world by gender in 5-year age groups.

The aim of the present report is to give an a priori description of the methods for the systematic review of the epidemiological literature on stroke incidence and casefatality. We will formally present the results of this process in a separate manuscript.

The objectives of the current manuscript are to: (1) establish and report stroke case-definitions; (2) identify measures of the clinical consequences of stroke; (3) document our systematic literature search strategy for retrieving data on key epidemiologic measures; (4) describe cri- teria for inclusion or exclusion of studies and other data sources identified in the review; (5) describe study coding procedures, data categories, and study quality measures for the systematic review; (6) provide a description of an analytic model for estimating the global epidemiology of stroke, and (7) describe statistical procedures for the quantitative analysis of data from eligible studies. Our overall objective is to present a transparent process for how the information will be collected on the global epidemiology of stroke based on explicit definitions of disease states and mortality risks, and summarize the techniques that will be used to maximize the validity of these measurements by addressing bias, confounding and missing data.

\section{Methods}

Stroke Definition and Pathogenesis

Stroke is defined according to the World Health Organization (WHO) criteria as 'rapidly developing clinical signs of focal (at times global) disturbance of cerebral function lasting more than $24 \mathrm{~h}$ or leading to death with no apparent cause other than that of vascular origin' [11]. This definition of stroke excludes neurological deficits from epidural and subdural haemorrhage, trauma, malignancy and infection-related cerebral haemorrhage or infarction, and transient ischaemic attacks (TIAs) characterized by brief episodes of focal loss of brain function of less than $24 \mathrm{~h}$ and with no persistent deficit. Recently, a proposal for a tissue-based definition of stroke and TIA, based on the results of neuroimaging, has been adopted by some research groups [12]. For the purposes of this updated GBD project, however, and to maintain consistency with previous GBD reports, we used the classic definition of TIA and stroke and applied the standard WHO definition of stroke for study inclusion as availability of neuroimaging (and type of neuroimaging) varies between and within regions (e.g. urban versus rural) of the world.

Pathological Types of Stroke

There are different pathological types of stroke: ischaemic stroke (IS), primary intracerebral haemorrhage (PICH), and subarachnoid haemorrhage (SAH). Population-based studies of European-descent populations from the United States and Europe estimate the distribution of stroke types to be approximately $73-$ 90\% IS, 9-13\% PICH, and 1-6\% SAH (in low- or middle-income countries, $54-85 \%, 14-27 \%$ and $5-10 \%$, respectively) $[4,13]$. Recent data from China show a pattern of a rising proportion of IS, and a falling proportion of haemorrhagic stroke, suggesting that regional differences in the proportion of stroke subtypes may be due to differences in economic development and risk factor profiles [14]. These observations underscore the importance of measuring pathological stroke subtypes.

Ischaemic Stroke

Cerebral ischaemia can be either global or focal. Specific arteries supply cerebral areas and a focal neurological deficit may reflect the location of the infarct. In other instances, a cerebrovas- 
cular lesion may not result in a clearly delineated acute syndrome, depending on the artery involved, collateral circulation, and variations in the region supplied by a particular artery [15]. Onemonth case-fatality for IS in different countries ranges from 13 to $23 \%[4]$.

\section{Cerebral and Subarachnoid Haemorrhage}

Cerebral haemorrhage can occur with haemorrhage into the brain parenchyma (intracerebral haemorrhage) or into the ventricles (intraventricular haemorrhage), and subarachnoid haemorrhage with haemorrhage into the subarachnoid space [13]. Among individuals less than 40 years of age, rupture of an aneurysm, arteriovenous malformation, or illicit drug use are causal factors. Between the ages of 40 and 69 years, hypertension is the predominant cause [16]. Among the elderly, the cause is often unknown and may result from arteriopathy such as amyloid angiopathy [17]. Cerebral haemorrhage is associated with high (25$35 \%)$ risk of early death (21 days to 1 month) [4].

Diagnosis of Acute Stroke and the Role of Neuroimaging and Cardiac Testing

The fundamental criterion for diagnosis of acute stroke is the detection of a sudden-onset focal neurological deficit. In an 'ideal' high-quality population-based stroke epidemiological study [18] primary stroke subtypes would be confirmed by computed tomography (CT), magnetic-resonance imaging (MRI), or autopsy findings for approximately $70 \%$ or more of stroke cases. If this is not possible, the clinical history obtained from a responsive and oriented patient and/or family members, together with physical examination, can help differentiate a stroke from other clinical conditions such as severe hypoglycaemia, anaemia, hypoxaemia, toxic or metabolic disorders. However, neuroimaging is necessary to differentiate ischaemia from haemorrhage as the cause of stroke. Among the neuroimaging modalities, non-contrast CT is the most widely available and highly sensitive test for excluding intracerebral haemorrhage in the acute stroke setting. Although widely available in most developed countries, CT is generally less readily available in developing countries, particularly in rural settings. Other commonly used imaging modalities in stroke patients include neurovascular imaging with MRI or CT angiography, and ultrasonography of the intracranial and extracranial vessels to identify large vessel sources of ischaemic stroke [19-21]. Electrocardiography, telemetry and echocardiography can be used to identify cardioembolic causes of ischaemic stroke.

\section{Natural History of Stroke}

Although the majority of stroke-related deaths occur beyond the hyperacute period [22] stroke can lead to sudden death (within $6 \mathrm{~h}$ of onset) [23] or death within $24 \mathrm{~h}$ of the onset of symptoms, especially when the associated cerebral ischaemia or brain haemorrhage is extensive and/or involves critical brain centres [23]. $\mathrm{PICH}$ and SAH are more likely to lead to sudden death than IS. Very sudden death (within $1 \mathrm{~h}$ of symptom onset), as opposed to sudden death over hours, is more often cardiac in origin than stroke in origin - a factor to consider in studies relying on verbal autopsy where stroke might be over diagnosed [24].

For persons who survive past $24 \mathrm{~h}$, the natural history of stroke in the short and long term (beyond 28 days) have been elucidated through several observational, community, and populationbased studies [25-31]. The 21-day to 1-month case fatality has been reported most recently as ranging from 13 to $23 \%$ for IS, as compared to $25-35 \%$ for ICH, $25-35 \%$ for SAH in high-income countries [4]. In general, advancing age, stroke severity, underlying heart disease (including atrial fibrillation), diabetes mellitus, pre-morbid disability/dependence and dementia and access to evidence-based therapies (thrombolysis and organized stroke rehabilitative care) are important determinants of stroke outcome (death and disability) [25, 26, 32-46].

\section{Stroke GBD Systematic Literature Review}

Study Eligibility

Manuscripts of studies conducted among any population throughout the world are potentially eligible for inclusion in the systematic review. For inclusion, the studies in high-income countries adhered to the following core requirements: (1) distinguished between stroke and transient ischaemic attack (TIA) based on a duration of symptomatic focal neurological deficit more (stroke) or less (TIA) than $24 \mathrm{~h}$; (2) distinguished between first-ever stroke and recurrent stroke; (3) reported on an age-specific epidemiologic parameter of interest (i.e. stroke incidence, prevalence, disease specific mortality, relative risk of mortality, case fatality) sufficient to estimate age-adjusted stroke incidence or prevalence; (4) described the method for estimating the actual age-specific value of the population denominator in communitybased studies; (5) incidence and 1-month case fatality studies from developed countries required a complete stroke case ascertainment (hospitalized and non-hospitalized, fatal and non-fatal cases) using multiple overlapping sources of information; (6) if reported, provided survival status within 28 days, 30 days or 1 month of onset for fatal and non-fatal events; (7) specified methods for ascertaining stroke cases diagnosed and/or treated outside the hospital, and (8) described and provided the proportion of cases where imaging modalities and diagnostic testing were used to determine stroke subtypes. Only stroke incidence and/or prevalence studies with age-specific data (no restrictions on age range) will be included in the analysis.

In contrast to earlier GBD reviews, we included studies from low- or middle-income countries with a relaxation of the aforementioned eligibility criteria used for high-income countries. For example, less rigorous stroke case ascertainment was only allowed for studies from low- or middle-income countries in which no other relevant data were available. These studies were scrutinized carefully for evidence of epidemiological rigour and the most methodologically reliable studies from such countries were given priority for calculating incidence and early case fatality (defined as 28-day, 30-day or 1-month, case fatality) estimates for the GBD region concerned.

Literature Search Strategy

We searched multiple databases (Medline, EMBASE, LILACS, Scopus, PubMed and Sciencedirect, Global Health Database [formerly CAB abstracts], WHO library and WHO regional databases) in order to identify studies published between 1980 and 2011. We searched the databases using the words: 'stroke', 'isch(a)emic stroke', 'intracerebral', 'intraparenchymal', 'subarachnoid', ' $\mathrm{h}(\mathrm{a})$ emorrhage' AND title or key-words 'population-based', 'community-based', 'community', 'epidemiology', 'epidemiological', 'prevalence' 'incidence', 'attack rates', 'survey', 'surveillance', 'mortality', 'morbidity', 'fatality', 'case-fatality', or 'trends'. (The full search strategy is available on request from the authors.) 


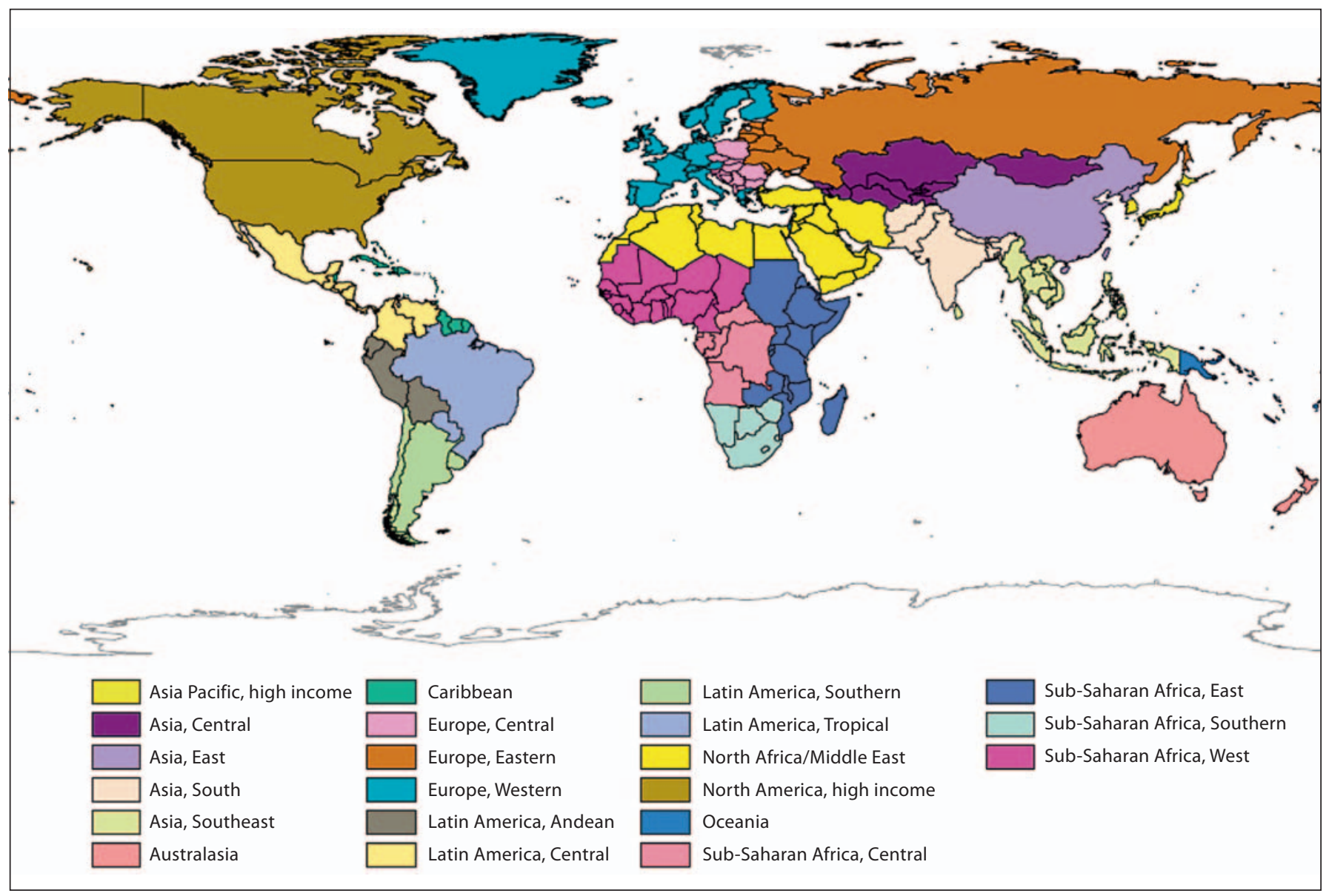

Fig. 1. Map of the 21 global burden of disease, injuries and risk factor regions.

The reliability of the literature search strategy was evaluated by comparing 50 key citations nominated by a group of stroke experts across GBD regions to the studies identified from the literature search in order to ascertain whether these citations were retrieved. The results of the systematic literature searches were stored in a bibliographic database. Multiple searches were screened for duplicate citations.

Study Identification

The criteria for relevance applied to the studies identified in the literature searches were assessed in two stages. The first stage of relevant decisions on eligibility was based on screening titles and abstracts. Specifically, titles and abstracts were included if they indicated that it was a population-based study examining incidence, prevalence, case fatality or mortality. This might include cohorts, cross-sectional surveys, disease registry, or notification data. Health facility data was included if they reported case fatality in a well-specified population or an entire region. The list of all titles and abstracts identified in stage one provided a 'pool' of the global epidemiological evidence-base for stroke. In the second stage, members of the review team identified all potential studies that met the eligibility criteria that provided the most relevant data for GBD estimates. Based on the results of the search strategy, members of the review team reviewed the titles, abstracts and key words of every report retrieved. Full reports of these citations were obtained for further assessment of the study for inclusion in the systematic review.

Data Extraction

Eligible studies included in the GBD stroke review were recorded and coded into a detailed electronic data extraction form (available on request from the authors) by trained study reviewers. In brief, the data extraction form contained information on The International Classification of Diseases-10 (ICD-10) codes relevant to IS and haemorrhagic stroke as well as details on age, ethnicity and gender groups. Information on region of the world where the study was conducted was recorded. The specific countries within each region are shown graphically in figure 1.

Study data were coded as urban, rural or combined. The scope of the epidemiologic study was classified as national, sub-national, healthcare system-based, or community level. Studies published in languages other than English or Spanish were translated 


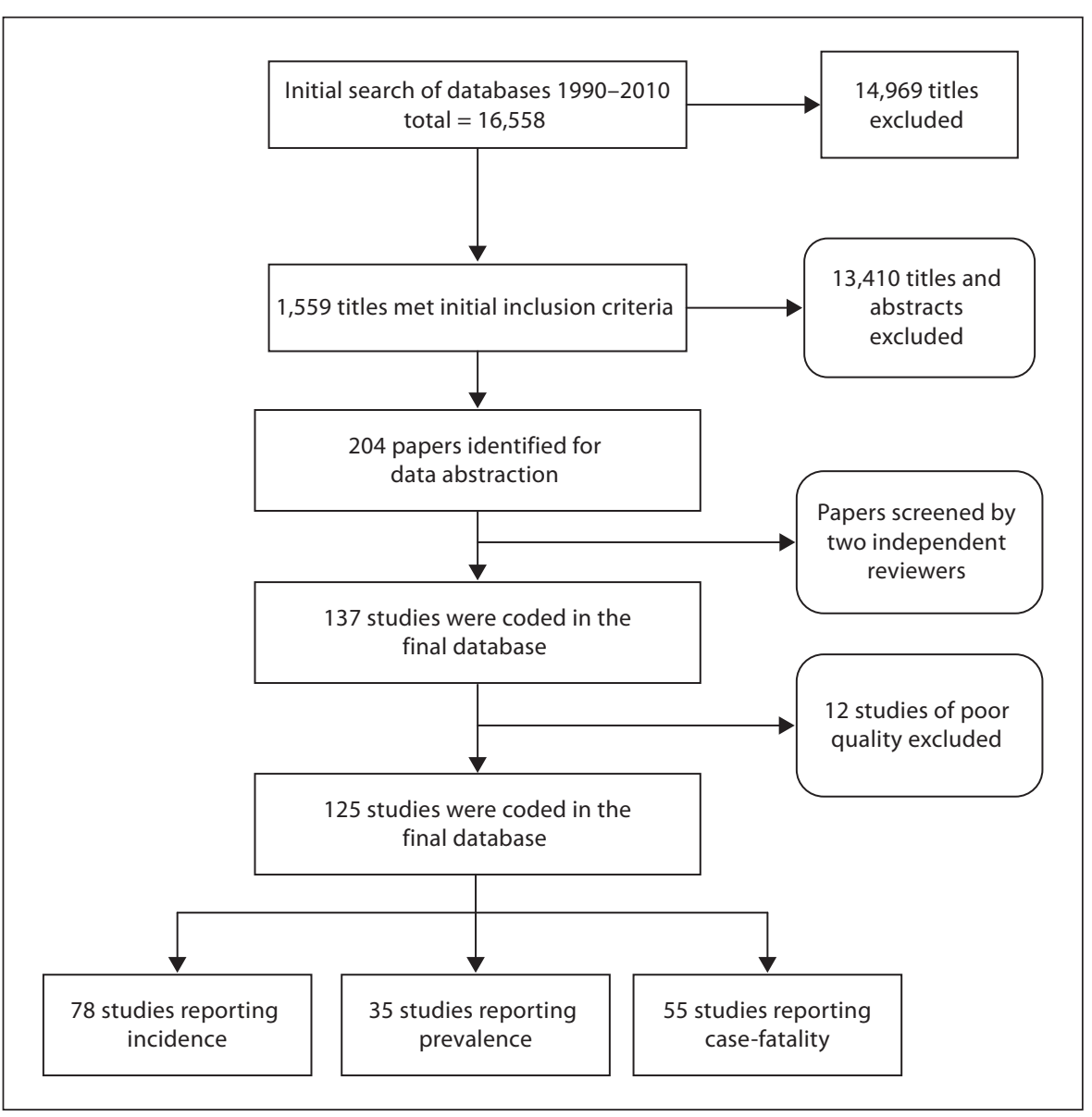

Fig. 2. Flowchart describing preliminary search results of the stroke GBD systematic review process.

ity of observational studies [47]. We separated out methodological

and coded centrally at the University of Washington Institute for Health Metrics and Evaluation. A 10\% subset of reports were independently selected by two reviewers for inclusion in the review from the list of potentially eligible studies and assessed for consistency. A flowchart detailing the eligible studies is described in figure 2 .

As a variety of study types and data sources were eligible from the stroke review literature, information was also be recorded on: the appropriateness of the particular study design to estimate relevant disease parameters; whether the data are representative of the population; the identification of possible sources of bias, and 'methodologically specific quality' and completeness of data reported. We discriminated between reporting and methodological quality. 'Methodologically specific quality' refers to the extent to which specific aspects of a study or data source can be shown to protect against systematic bias, non-systematic bias, and inferential error for the disease parameter to be estimated in the GBD model. An outline of the 'methodologically-specific quality' issues that were recorded in the data extraction form that pertain to different disease parameters are given in table 1 for each study type.

A ten item checklist was included in the study coding form to assess general reporting quality of the study. The ten items were derived from those included in the Strengthening the Reporting of Observational Epidemiology statement for assessing the qual- quality from general reporting quality as it is important to clarify and differentiate between quality of reporting and the quality of what was actually done (i.e. a study could be well reported but have methodological limitations) $[48,49]$. The collection of this general reporting quality information as well as 'methodologically specific' quality information enables the exploration of possible sources of heterogeneity and can also be used to conduct sensitivity analyses in order to quantify the magnitude of possible bias in study estimates based on particular characteristics of the study. Sensitivity analyses will be based on stratification by individual items of 'methodologically specific' or (where appropriate), individual items of general reporting quality in order to assess the robustness of the findings. The included items for general reporting quality are shown in table 2.

Synthesis of Individual Estimates

The individual study estimates from our systematic review will be combined using inverse-variance weighting techniques [50] in order to provide information on the relevant estimates of incidence, prevalence and other useful parameters that will then be used in the stroke-specific GBD modelling analyses. This implies that larger studies will be given more weight in the analyses when producing an overall pooled estimate for a particular GBD
The Global Burden of Disease Stroke Expert Group 
Table 1. Methodologically specific quality

\begin{tabular}{lll}
\hline Parameter & Study types & Appraisal issues \\
\hline Incidence & $\begin{array}{l}\text { disease registers } \\
\text { cohort studies } \\
\text { notification data } \\
\text { hospital data }\end{array}$ & $\begin{array}{l}\text { completeness } \\
\text { representative } \\
\text { validity of case ascertainment }\end{array}$ \\
\hline Prevalence & national surveys & representative \\
& local epidemiological studies & validity of case ascertainment \\
\hline Mortality and case fatality & disease registers & $\begin{array}{l}\text { cases studied representative for all cases } \\
\text { completeness } \\
\end{array}$ \\
& $\begin{array}{l}\text { hospital data } \\
\text { national vital statistics } \\
\text { cohort studies }\end{array}$ & $\begin{array}{l}\text { representative } \\
\end{array}$ \\
\hline
\end{tabular}

Table 2. General reporting quality assessment items

1 Study design and methods are appropriate for the research question.

2 Sampling frame is appropriate for estimating the epidemiologic measures of interest.

3 Use WHO stroke definition 'rapidly developing clinical signs of focal (or global) disturbance of cerebral function, with symptoms lasting $24 \mathrm{~h}$ or longer or leading to death, with no apparent cause other than of vascular origin'.

4 Sample size is adequate for robust estimates.

5 Denominator data are reliable and sources documented.

6 Confidence intervals or variance estimates are reported.

7 Sources of potential bias are adequately controlled.

8 Study limitations that can influence interpretation of findings are identified.

9 The study methodology was sufficiently rigorous to support the conclusion of this study.

10 Study subjects and setting are similar to those for whom the results are generalised.

All of these items are coded as yes/no.

region. This approach is more robust to small study biases (of which publication bias is just one aspect of this umbrella term) [51].

\section{Generic GBD Analytic Model}

The GBD Study seeks to achieve internal consistency of epidemiological profiles for each disease and their associated disabling sequelae. Experience from the original GBD study showed that epidemiological estimates did not have sufficient internally con-

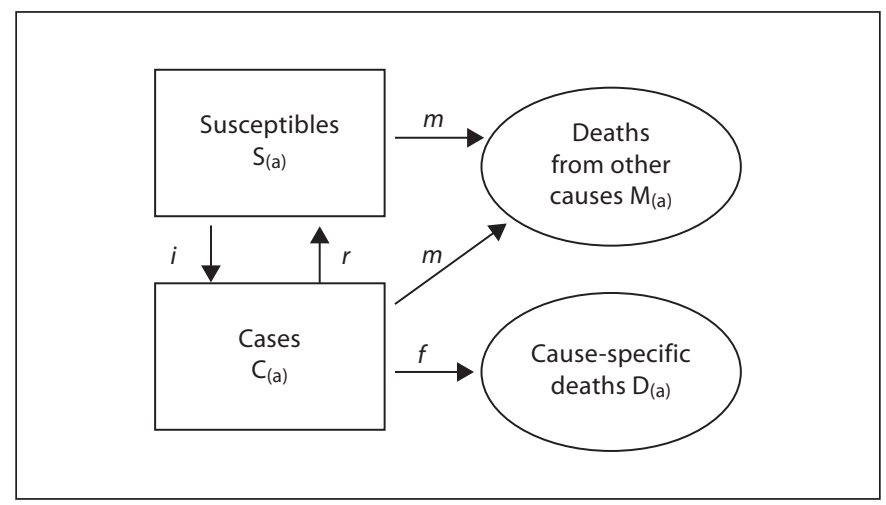

Fig. 3. Basic relationship between susceptibles, cases and deaths.

sistent, partly because different epidemiologic parameters come from different studies or age groups [10]. Parameters such as estimated prevalence were not always consistent with estimated incidence (e.g. because survivors may age and hence current prevalence may reflect past incidence), nor were case-fatality data consistent with death data. In an effort to improve internal consistency, a generic model formalizing the relationship between susceptible population, cases and deaths was developed [52] for use in GBD studies and is illustrated in figure 3.

The software program DisMod III (that uses a unique compartmental model to model the data with similar parameters but different assumptions to those used in DisMod II [52]) was developed for the GBD to model these parameters. Models are based on a user specified set of age-specific prevalence, incidence, remission and case-fatality for a specific disease, region and gender. In the generic GBD model that uses DisMod, remission was defined as a complete cure (i.e. an individual can revert from being a case to being non-case and rejoin the susceptible population). Susceptible individuals in the population, denoted as $S_{(a)}$ in the 


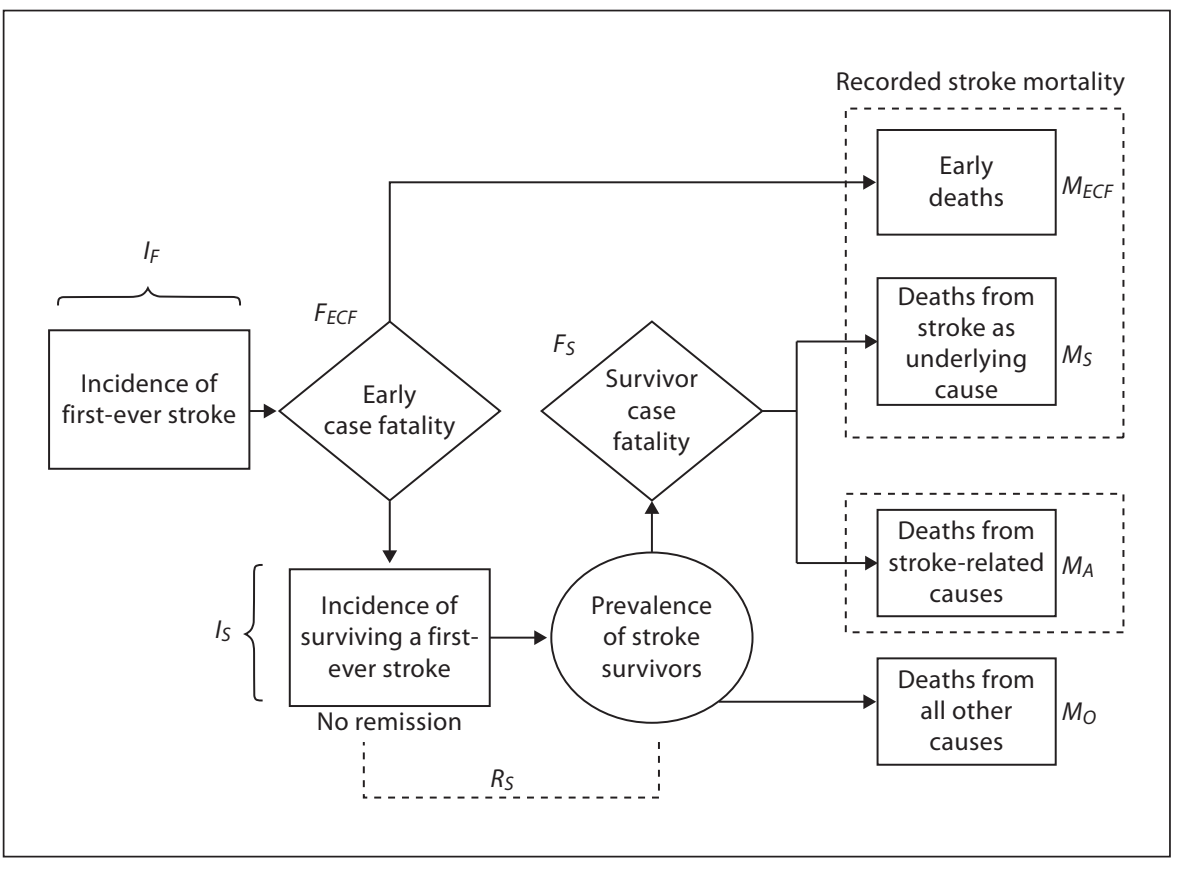

Fig. 4. Schematic diagram for estimating the global burden of stroke.

model, are at risk of incurring a disease or disability at rate $i$ and can die at a general mortality rate $m$. Cases of disease, denoted as $C_{(a)}$, can experience remission at rate $r$, die at the same rate as the susceptible individuals $m$, or die from case-specific mortality from a condition at rate $f$. Within a particular user defined age interval, expressed as a in the model, rates $\mathrm{i}, \mathrm{r}$ and $\mathrm{f}$ can be estimated. If the user provides information on various mortality measures (e.g. relative risk or standardized mortality ratio) then DisMod III can use this information to estimate an appropriate mortality measure such as excess mortality.

\section{Stroke-Specific GBD Model}

To identify inconsistent estimates and modify them to be more consistent, a stroke-specific model formalizing the relationship between stroke prevalence, incidence, remission, and case-fatality was developed and is presented in figure 4 [53].

The model assumes that, for ischaemic and haemorrhagic stroke that first ever stroke is being estimated. For the purposes of modelling, total stroke estimates will be used as the upper boundary for the summation of ischaemic and haemorrhagic estimates. To employ this assumption, data for 'total stroke' will be modelled separately to produce overall estimates of incidence $\times$ duration. This is because burden is measured as an event-based metric so this measure is required in order for there to be consistency between fatal (life lost to premature death) and non-fatal (healthy life years lost due to incident stroke). Once an individual has a stroke, they cannot return to the susceptible population. Therefore, a zero remission rate will be assumed for all stroke GBD models. Although individuals may improve back to a baseline health state, this change will be reflected in an improved disability weight and not treated as a remission of the underlying condition.
The model also will assume that in countries with good vital registration, all deaths within 1 month of a stroke event are certified as stroke deaths. '28-day', '30-day' and '1-month case fatality' will be assumed to be sufficiently similar to group together to act as with-condition mortality rates. We will use the term 'early case-fatality' to cover '28-day', '30-day' and '1-month' case-fatality.

The total number of deaths due to stroke in the country's vital register can be defined as:

$$
M_{C}=M_{E C F}+M_{S}
$$

where $M_{E C F}$ is the number of stroke deaths (i.e. within, 28-days, 30 -days or 1-month of a stroke) and $M_{S}$ is the number of deaths in which stroke is identified as the underlying cause of death in 28-day, 30-day or 1-month survivors.

Since $M_{E C F}$ is a product of the hazard of dying within 28-days, 30-days or 1-month, $M_{s}$ can be expressed as:

$$
M_{S}=M_{C}-I_{F} F_{E F C}
$$

where $I_{F}$ is the incidence of first ever stroke and $F_{E C F}$ is the early case fatality.

The remaining mortality in survivors can be divided into deaths due to causes closely related to stroke $M_{A}$ (e.g. brain oedema, other cardiovascular disease) and deaths due to causes unrelated to stroke $M_{O}$. Although $M_{A}$ cannot be computed directly, it can be derived indirectly via DisMoD III as it can compute excess mortality $\left(M_{A}+M_{O}\right)$ from inputs such as relative risks or standardized mortality ratios. DisMod III can calculate an internally consistent description of disease epidemiology from at least three known parameters related to stroke. Permitted parameters include: incidence rate, prevalence rate, duration of survival, relative risk of mortality, remission and case fatality. 
Table 3. Sequelae of stroke

\begin{tabular}{|c|c|}
\hline Sequelae & Definition \\
\hline $\begin{array}{l}\text { Ischaemic } \\
\text { stroke }\end{array}$ & $\begin{array}{l}\text { Cases of stroke according to WHO definition } \\
\text { (excludes transient ischaemic attacks and haem- } \\
\text { orrhage or infarction due to infection or tumour). }\end{array}$ \\
\hline $\begin{array}{l}\text { Haemorrhagic } \\
\text { and other } \\
\text { non-ischaemic } \\
\text { stroke }\end{array}$ & $\begin{array}{l}\text { Cases of stroke according to WHO definition (in- } \\
\text { cludes subarachnoid haemorrhage but excludes } \\
\text { transient ischaemic attacks, subdural haemato- } \\
\text { ma, and haemorrhage or infarction due to infec- } \\
\text { tion or tumour) excluding ischaemic stroke. }\end{array}$ \\
\hline $\begin{array}{l}\text { First-ever } \\
\text { stroke in } \\
\text { a lifetime }\end{array}$ & $\begin{array}{l}\text { Visual and other sensorimotor deficits, unable to } \\
\text { care for oneself or perform activities of daily liv- } \\
\text { ing, severe mobility limitation, likely cognitive } \\
\text { and motor deficits. The average duration of these } \\
\text { deficits is } 6 \text { days for those early case fatalities. } \\
\text { This health state will be modelled with duration } \\
\text { of } 6 \text { days for all 1st strokes [based on stroke expert } \\
\text { panel consensus]. }\end{array}$ \\
\hline $\begin{array}{l}28 \text {-day } \\
\text { survivor }\end{array}$ & $\begin{array}{l}\text { Individuals that survive for at least } 28 \text { days after } \\
\text { first-ever stroke are estimated from incidence of } \\
\text { first-ever stroke and measured } 28 \text {-day case-fatal- } \\
\text { ity rate. The stroke disease model assumes as an } \\
\text { initial estimate that } 70 \% \text { of long-term survivors } \\
\text { of stroke have long-term disability [based on } \\
\text { stroke expert panel consensus]. }\end{array}$ \\
\hline
\end{tabular}

To produce estimates for the incidence of chronic stroke survivorship, we will assume that the (incidence of acute stroke) $\times$ (early case survival) will be a plausible value.

Certain parameter types may not be used in the analysis because they cannot be converted into usable DisMod III parameters. Examples include 1-week case fatality, 3-month case fatality or severity indices. Cause specific mortality will be used to establish a lower limit that the estimate of excess mortality $\times$ prevalence was not permitted to go below.

A difficulty with individual estimates of ischaemic and haemorrhagic stroke is that there is likely to be minimal prevalence data available that categorizes chronic stroke survivors by type. However, a recent report by Katzenellenbogen et al. [54] has demonstrated how to estimate early case-fatality risk for HS and IS in a population from Western Australia that used a data linkage system that was able to probabilistically match data from death registrations and public and private hospitalisations. We will supplement this information with evidence from the systematic review of the literature, as well as via discussion with members of the stroke expert group. As a sensitivity analysis we assumed that in both of these chronic survivor populations, the relative risk of mortality was equal. This assumption allows the use of relative risk estimations produced for chronic total stroke to be used as an input into the ischaemic and haemorrhagic stroke models. In terms of the DisMod III modelling, it was assumed that relevant strokes occurred after the age of 10 . Those events occurring before that age are considered secondary, due to different aetiologies compared to those in the older population.

\section{Stroke Sequelae Modelling}

The GBD generic model links loss of health to disease and injury causes through the concepts of cases and sequelae. For incident cases of a given disease or injury, there will be a distribution of current and future health states which reflect the experience of health until remission or death for each incident case. The generic GBD model maps the distribution of health states for any disease or injury to a small set of discrete entities for which epidemiological estimates and years lost to disability calculations are made. The GBD Study uses the term 'sequelae' as an umbrella term to describe this set of discrete entities [55]. Depending on the nature of the disease, sequelae may include only the disease case, disease case and sequelae, or only disabling sequelae. For the stroke GBD analyses, we will model stroke sequelae using a combination of incidence and prevalence of early case fatality, incidence and prevalence of survival, as well as excess mortality. Incidence and prevalence for early case fatality will be estimated for those individuals with fatal stroke in the first 28 days. Incidence and prevalence of survival will be estimated separately for those that survive the first 28 days.

Table 3 outlines the definitions and sequelae that will be considered in the current stroke GBD analyses. We are aware that this list is not exhaustive, but we will use this restricted list for global population-based analyses because the aim is to measure overall burden in diverse populations, many with limited detail on clinical data. Where studies that meet the eligibility criteria for the systematic review have reported levels of disability using well recognised scales of measurement we will pool the estimates using inverse-variance weighting methods and will use this information in a sensitivity analysis in order to revise our initial estimates.

Table 4 outlines the definitions and disability-related sequelae of stroke that will be considered in the current GBD stroke analyses.

We will collect information on levels of disability from eligible studies that report appropriate measures of disability. An important distinction about DALY weights are that they are derived across a range of diseases (not disease specific). However, stroke specific scales can be mapped to DALYs or any equivalent functional outcome scale if the study design is appropriate [56]. We will aim to quantify disability weights based on reaching a consensus from members of stroke expert panel and by reviewing the current evidence.

\section{Discussion}

By the end of the first quarter of 2012 using the 'raw' data collected during the systematic reviews, estimates will be derived for 21 GBD world regions by age and sex with uncertainty intervals. This will require a number of imputations to deal with missing values and to check available estimates, for specific diseases, for internal consistency. DisMod III software will be used in order to (a) 
Table 4. Disability-related sequelae of stroke

\begin{tabular}{lll}
\hline GBD Code & Sequelae & Definition \\
\hline SS66 & consequences of stroke, mild & this person walks with a limp and has weakness in one hand \\
\hline SS67 & consequences of stroke, moderate & $\begin{array}{l}\text { this person has difficulty with walking, speaking and personal care; } \\
\text { the person is sometimes forgetful and confused }\end{array}$ \\
\hline SS68 & consequences of stroke, severe & $\begin{array}{l}\text { this person is confined to bed or a wheelchair, has difficulty speaking and } \\
\text { depends on others for feeding, toileting and dressing }\end{array}$ \\
\hline SS239 & consequences of stroke, moderate plus & $\begin{array}{l}\text { same as consequences of moderate stroke; in addition, this person has some } \\
\text { difficulty in moving around, in using the hands for lifting and holding things }\end{array}$ \\
\hline SS240 & consequences of stroke, severe plus & $\begin{array}{l}\text { same as consequences of stroke severe but additionally has difficulty speaking, } \\
\text { thinking clearly and remembering things }\end{array}$ \\
\hline
\end{tabular}

determine common age patterns, and (b) impute regional estimates from the data supplied by the systematic reviews. DisMod III can perform checks of internal consistency between the available disease parameters and, finally produce an internally consistent full set of epidemiological parameters describing a disease and its disabling sequelae.

As in previous GBD studies each step of estimating the stroke burden involves making informed assumptions such as length of time for survival, the survival rate in stroke survivors, survival distribution according to age and sex, and the proportion of subjects who died due to stroke or other causes. In order to enhance transparency a complete description of the methodology to be employed in deriving these estimates is essential, which is the purpose of this methodological report. Ideally, our estimates can be derived from comparable data from all 21 GBD regions about stroke occurrence, case-fatality rates, survival rates with and without disability, and other important outcomes. The quality of reporting, and the amount of information available from, low- and middle-income countries has improved since the previous stroke burden projections and this, in turn, will lead to more robust estimates for those regions where data were once very sparse. The recent growth and investment in the area of international health has made the need for an accurate assessment of global health patterns a matter of paramount importance. A thorough GBD reassessment of stroke and other disease and injury areas will ensure that the global health community bases policies and intervention strategies on the most up-to-date, evidence-based, valid and reliable epidemiological information as possible.

\section{Acknowledgements}

The authors would like to thank Colin Mathers and Stephen Begg who devised the modelling approach for the 2000 update of Global Burden of Disease.

The authors acknowledge the valuable contribution of Sara Safranek, University of Washington Health Sciences Library, who developed the systematic review literature search strategies.

Support from the Division of Heart Disease and Stroke Prevention, US Centers for Disease Control and Prevention, was provided for the participation of Laurie M. Anderson.

\section{Appendix}

Writing committee of the Global Burden of Disease Stroke Expert Group: Derrick A. Bennett, Oxford (UK), Laurie M. Anderson, Nathan Nair, Thomas Truelsen, Suzanne Barker-Collo, Myles Connor, Hannah Gardener, Rita Krishnamurthi, Carlene M.M. Lawes, Andrew Moran, Martin O’Donnell, Varsha Parag, Ralph L. Sacco, Majid Ezzati, George Mensah, and Valery L. Feigin.

References

The Global Burden of Disease Stroke Expert Group 
-4 Feigin VL, Lawes CM, Bennett DA, BarkerCollo SL, Parag V: Worldwide stroke incidence and early case fatality reported in 56 population-based studies: a systematic review. Lancet Neurol 2009;8:355-369.

5 Truelsen T, Bonita R, Jamrozik K: Surveillance of stroke: a global perspective. Int J Epidemiol 2001;30(suppl 1):S11-S16.

$\checkmark 6$ Beaglehole R, Saracci R, Panico S: Cardiovascular diseases: causes, surveillance and prevention. Int J Epidemiol 2001;30(suppl 1):S1-S4.

7 Murray CJL, Lopez AD: The Global Burden of Disease: A Comprehensive Assessment of Mortality and Disability from Diseases, Injuries, and Risk Factors in 1990 and Projected to 2020. Cambridge, Harvard University Press, 1996.

8 Mathers CD, Lopez AD, Murray CJL: The Burden of Disease and Mortality by Condition, Data, Methods, and Results for 2001. Global Burden of Disease and Risk Factors. Oxford University Press and The World Bank, 2006.

>9 Murray CJ, Lopez AD, Black R, et al: Global burden of disease 2005: call for collaborators. Lancet 2007;370:109-110.

10 Murray CJL, Lopez AD: Global and Regional Descriptive Epidemiology of Disability: Incidence, Prevalence, Heath Expectancies and Years Lived with Disability: The Global Burden of Disease. Cambridge, Harvard University Press, 1996, pp 201-246.

- 11 Hatano S: Experience from a multicentre stroke register: a preliminary report. Bull World Health Organ 1976;54:541-553.

-12 Albers GW, Caplan LR, Easton JD, et al: Transient ischemic attack - proposal for a new definition. N Engl J Med 2002;347:17131716.

13 Warlow CP, Dennis MS, van Gijn J, et al: What caused this transient or persisting ischemic event?; in Warlow CP, Dennis MS, van Gijn J, et al (eds): Stroke: A Practical Guide to Management, ed 1. Oxford, Blackwell Science, 2001, pp 223-300.

14 Zhao D, Liu J, Wang W, et al: Epidemiological transition of stroke in China: twentyone-year observational study from the SinoMONICA-Beijing Project. Stroke 2008;39: 1668-1674.

15 de Freitas GR, Bogousslavsky J: Ischemic stroke syndromes: clinical features, anatomy, vascular territories, and prognosis; in Adams HP (ed): Handbook of Cerebrovascular Diseases, ed 2. New York, Marcel Dekker, 2005, pp 43-71.

16 Lawes CM, Bennett DA, Feigin VL, Rodgers A: Blood pressure and stroke: an overview of published reviews. Stroke 2004;35:1024.

17 Chang K-C: Intracerebral hemorrhage; in Adams HP (ed): Handbook of Cerebrovascular Diseases, ed 2. New York, Marcel Dekker, 2005, pp 73-80.
18 Malmgren R, Warlow C, Bamford J, Sandercock P: Geographical and secular trends in stroke incidence. Lancet 1987;ii:1196-1200.

19 Lovblad KO, Altrichter S, Viallon M, et al: Neuro-imaging of cerebral ischemic stroke. J Neuroradiol 2008;35:197-209.

20 Lovblad KO, Baird AE: Actual diagnostic approach to the acute stroke patient. Eur Radiol 2006;16:1253-1269.

21 Castillo PR, Miller DA, Meschia JF: Choice of neuroimaging in perioperative acute stroke management. Neurol Clin 2006;24: 807-820.

22 Bamford J, Dennis M, Sandercock P, Burn J, Warlow C: The frequency, causes and timing of death within 30 days of a first stroke: the Oxfordshire Community Stroke Project. J Neurol Neurosurg Psychiatry 1990;53:824829.

23 Phillips LH, Whisnant JP, Reagan TJ: Sudden death from stroke. Stroke 1977;8:392395.

24 Thomas AC, Knapman PA, Krikler DM, Davies MJ: Community study of the causes of 'natural' sudden death. BMJ 1988;297:14531456.

25 Asplund K, Hagg E, Helmers C, Lithner F, Strand T, Wester PO: The natural history of stroke in diabetic patients. Acta Med Scand 1980;207:417-424.

26 Sacco RL, Wolf PA, Kannel WB, McNamara PM: Survival and recurrence following stroke: the Framingham study. Stroke 1982; 13:290-295.

27 Bamford J, Sandercock P, Jones L, Warlow C: The natural history of lacunar infarction: the Oxfordshire Community Stroke Project. Stroke 1987;18:545-551.

28 Barer DH: The natural history and functional consequences of dysphagia after hemispheric stroke. J Neurol Neurosurg Psychiatry 1989;52:236-241.

29 Patel M, Coshall C, Rudd AG, Wolfe CD: Natural history of cognitive impairment after stroke and factors associated with its recovery. Clin Rehabil 2003;17:158-166.

30 Paul SL, Dewey HM, Sturm JW, Macdonell RA, Thrift AG: Prevalence of depression and use of antidepressant medication at 5-years poststroke in the North East Melbourne Stroke Incidence Study. Stroke 2006;37: 2854-2855.

31 Haacke C, Althaus A, Spottke A, Siebert U, Back T, Dodel R: Long-term outcome after stroke: evaluating health-related quality of life using utility measurements. Stroke 2006; 37:193-198.

32 Appelros P, Nydevik I, Viitanen M: Poor outcome after first-ever stroke: predictors for death, dependency, and recurrent stroke within the first year. Stroke 2003;34:122126.

33 Sturm JW, Donnan GA, Dewey HM, et al: Quality of life after stroke: the North East Melbourne Stroke Incidence Study (NEMESIS). Stroke 2004;35:2340-2345.
34 Jorgensen HS, Reith J, Nakayama H, Kammersgaard LP, Raaschou HO, Olsen TS: What determines good recovery in patients with the most severe strokes? The Copenhagen Stroke Study. Stroke 1999;30:2008-2012.

-35 Varona JF, Bermejo F, Guerra JM, Molina JA: Long-term prognosis of ischemic stroke in young adults: study of 272 cases. J Neurol 2004;251:1507-1514.

-36 Srikanth VK, Quinn SJ, Donnan GA, Saling MM, Thrift AG: Long-term cognitive transitions, rates of cognitive change, and predictors of incident dementia in a populationbased first-ever stroke cohort. Stroke 2006; 37:2479-2483.

37 Vemmos KN, Bots ML, Tsibouris PK, et al: Prognosis of stroke in the south of Greece: 1 year mortality, functional outcome and its determinants: the Arcadia Stroke Registry. J Neurol Neurosurg Psychiatry 2000;69:5955600 .

38 Indredavik B, Rohweder G, Naalsund E, Lydersen S: Medical complications in a comprehensive stroke unit and an early supported discharge service. Stroke 2008;39:414420.

39 Rocco A, Pasquini M, Cecconi E, et al: Monitoring after the acute stage of stroke: a prospective study. Stroke 2007;38:1225-1228.

$\checkmark 40$ McLean DE: Medical complications experienced by a cohort of stroke survivors during inpatient, tertiary-level stroke rehabilitation. Arch Phys Med Rehabil 2004;85:466469.

41 Roth EJ, Lovell L, Harvey RL, Heinemann AW, Semik P, Diaz S: Incidence of and risk factors for medical complications during stroke rehabilitation. Stroke 2001;32:523529.

42 Langhorne P, Stott DJ, Robertson L, et al: Medical complications after stroke: a multicenter study. Stroke 2000;31:1223-1229.

43 Davenport RJ, Dennis MS, Wellwood I, Warlow CP: Complications after acute stroke. Stroke 1996;27:415-420.

44 Erhan H, Ochoa E, Borod J, Feinberg T: Consequences of right cerebrovascular accident on emotional functioning: diagnostic and treatment implications. CNS Spectr 2000;5: 25-38.

45 Feigin VL, Barker-Collo S, Parag V, et al: Auckland Stroke Outcomes Study. 1. Gender, stroke types, ethnicity, and functional outcomes 5 years poststroke. Neurology 2010;75:1597-1607.

$\checkmark 46$ Barker-Collo S, Feigin VL, Parag V, Lawes CM, Senior H: Auckland Stroke Outcomes Study. 2. Cognition and functional outcomes 5 years poststroke. Neurology 2010;75:16081616. 
-47 von Elm E, Altman DG, Egger M, Pocock SJ, Gotzsche PC, Vandenbroucke JP: The Strengthening the Reporting of Observational Studies in Epidemiology (STROBE) statement: guidelines for reporting of observational studies. Internist (Berl) 2008;49: 688-693.

48 Sanderson S, Tatt ID, Higgins JP: Tools for assessing quality and susceptibility to bias in observational studies in epidemiology: a systematic review and annotated bibliography. Int J Epidemiol 2007;36:666-676.
49 Shamliyan T, Kane RL, Dickinson S: A systematic review of tools used to assess the quality of observational studies that examine incidence or prevalence and risk factors for diseases. J Clin Epidemiol 2010;63:10611070.

50 Hedges L, Olkin I: Statistical Methods for Meta-analysis. San Diego, Academic Press, 1985.

51 Sterne JA, Egger M, Smith GD: Systematic reviews in health care: Investigating and dealing with publication and other biases in meta-analysis. BMJ 2001;323:101-105.

-52 Barendregt JJ, Van Oortmarssen GJ, Vos T, Murray CJ: A generic model for the assessment of disease epidemiology: the computational basis of DisMod II. Popul Health Metr 2003;1:4.
53 Truelsen T, Begg S, Mathers C: Global Burden of Cerebrovascular Disease in the Year 2000. Geneva, World Health Organization, 2002.

54 Katzenellenbogen JM, Begg S, Somerford P, et al: Disability burden due to stroke in Western Australia: new insights from linked data sources. Int J Stroke 2010;5:269-277.

55 Institute for Health Metrics and Evaluation University of Washington: Global Burden of Diseases, Injuries and Risk Factors Study Operations Manual, 2009.

56 Post PN, Stiggelbout AM, Wakker PP: The utility of health states after stroke: a systematic review of the literature. Stroke 2001;32: 1425-1429. 